\title{
Mathematical model of checkweigher with electromagnetic force balance system
}

\author{
Yuji Yamakawa ${ }^{1}$, Takanori Yamazaki ${ }^{2}$ \\ ${ }^{1}$ University of Tokyo, Hongo 7-3-1, Bunkyo-ku, Tokyo, Japan \\ ${ }^{2}$ Tokyo Denki University, Hatoyama, Hiki-gun, Saitama, Japan
}

\begin{abstract}
Our aim was to develop a high-speed and high-accuracy mass measurement system to be used with a conveyor belt (a checkweigher). The objective in this paper was to present a dynamic model of our proposed measurement system. The checkweigher with an electromagnetic force balance system was approximated using a spring-mass-damper system as the physical model, and the equation of motion was derived. The model parameters could be obtained from experimental data. Finally, the validity of the proposed model was confirmed by comparison of the simulation results with the experimental responses. The dynamic model obtained offers practical and useful information to examine the control scheme and to achieve high-performance mass measurement.
\end{abstract}

Section: RESEARCH PAPER

Keywords: mass measurement; electromagnetic force balance system; dynamic behavior

Citation: Yuji Yamakawa, Takanori Yamazaki, Mathematical model of checkweigher with electromagnetic force balance system, Acta IMEKO, vol. 3, no. 2, article 4, June 2014, identifier: IMEKO-ACTA-03 (2014)-02-04

Editor: Paolo Carbone, University of Perugia

Received March $4^{\text {th }}, 2013$; In final form August $14^{\text {th }}, 2013$; Published June 2014

Copyright: (C) 2014 IMEKO. This is an open-access article distributed under the terms of the Creative Commons Attribution 3.0 License, which permits unrestricted use, distribution, and reproduction in any medium, provided the original author and source are credited

Funding: (none reported)

Corresponding author: Yuji Yamakawa, e-mail:Yuji_Yamakawa@ipc.i.u-tokyo.ac.jp

\section{INTRODUCTION}

Recently, highly accurate mass measurement systems for packages moving along a high-speed conveyor belt — so-called "checkweighers" - have been increasing in importance in the food and manufacturing logistics industries. To achieve highspeed (continuous) measurement, packages should be moved in sequence. This means that the measuring time for each package is very short. In the near future, continuous mass measurement for 300 products per minute will be required.

In general, there are two types of checkweigher systems, namely the load-cell type and the electromagnetic force compensation (EMFC) type. In the load-cell system, the mass of an object can be measured by the deformation of the Roberval mechanism when the mass to be measured is put on a weighing pan. Although this type is applicable to a wide range of masses, it has difficulty achieving high accuracy. On the other hand, the EMFC system keeps a balance with the displacement of the lever-linked Roberval mechanism with electromagnetic force. Then, the mass of the object can be measured by the driving (or feedback) current for the electromagnetic force. This type has achieved high accuracy by the null method, but is limited in its range of measurement.
Ono, W. G. Lee, and Kameoka have already clarified in detail the dynamic behavior of the load-cell system [1]-[4]. But the dynamic behavior of the EMFC system has not yet been presented. Our aim was to improve the performance of the checkweigher for better speed and accuracy. To do this, it was necessary to develop a control scheme for the EMFC. We introduced a dynamic model of our proposed checkweigher using EMFC in previous papers [5]. However, the validity of the model for the closed-loop system could not be confirmed. In this paper, the model of the measurement system is improved. Then, the validity of this model is confirmed by a comparison with actual experimental results.

\section{MEASUREMENT SYSTEM}

Figure 1 shows the overall scheme of the checkweigher. Only a measuring conveyor appears in the photograph; the feed conveyor is located to the left of the measuring conveyor and the sorter is located to the right. The products are moved by the feed conveyor, the mass of product measured by measuring conveyor and the product is removed by a sorter outside of the measurable range.

The enlarged photograph of the mass measurement 


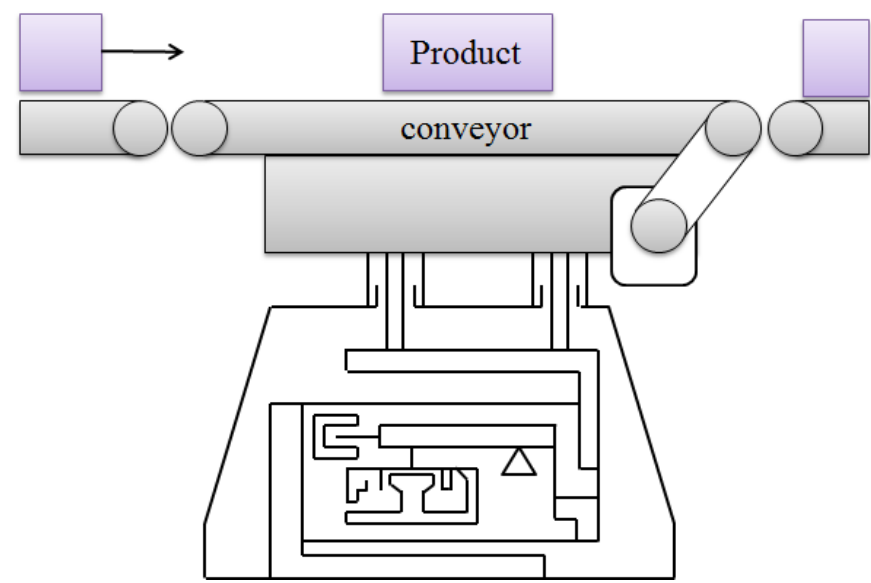

Figure 1. Overall of checkweigher.

mechanism in the checkweigher is shown in Figure 2. The mass measurement system consists of a weighing platform, the Roberval mechanism, the lever-linked Roberval mechanism, the counter weight, the electromagnetic force actuator and the displacement sensor. By applying the Roberval mechanism to the measurement mechanism, the mass of the product can be measured regardless of where the product is located on the weighing platform.

The mass of the product is estimated from the current of the electromagnetic force actuator to control the lever displacement.

The mass-measuring method can be explained as follows:

1. When the product of the mass $M$ is put on the measurement system, this causes the displacement of the Roberval mechanism.

2. The displacement of the Roberval mechanism is magnified twenty times by the lever.

3. The magnified displacement can be measured by the displacement sensor.

4. The current is controlled so that the displacement of the lever is maintained at zero.

5. The current is measured and the current is converted to the estimated mass using a linear function.

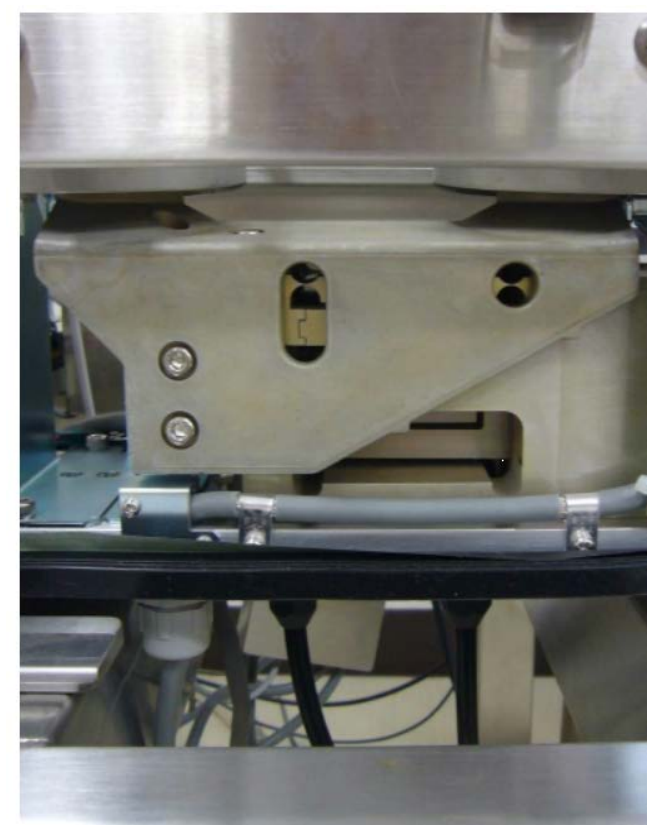

Figure 2. Photograph of Roberval mechanism.

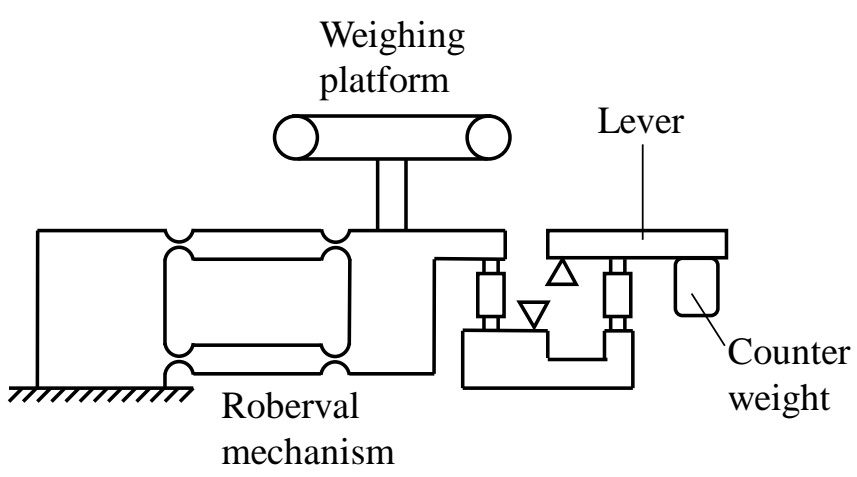

Figure 3. Overall of measurement system.

\section{MODELING}

Figure 3 and Figure 4 show the physical model of the measurement system. The equation of motion about mass $m$ can be obtained as follows:

$(\boldsymbol{M}+\boldsymbol{m}) \overline{\mathbf{x}}+\boldsymbol{m}_{\mathbf{L}} L^{2} \overline{\mathrm{x}}+\boldsymbol{c} \overline{\mathrm{x}}+\boldsymbol{k} \boldsymbol{\alpha}=\boldsymbol{M g}+\boldsymbol{F L}$,

where $m$ is the mass of the Roberval mechanism, $M$ is the mass of the product to be measured, $c$ is the a damping coefficient, $k$ is a spring constant, $g$ is the acceleration of gravity, $L(=20$ $\mathrm{m} / \mathrm{m}$ ) is the lever ratio, and $x$ is the displacement of mass $\mathrm{m}$. In addition, $m_{L}$ is the mass of the lever, $x_{L}$ is the displacement of mass $m_{L}$, and $F(=B l i$, where $B$ is a density of magnetic flux, $l$ a length of the coil, and $i$ a current) is an electromagnetic force input to control the position $x_{L}$ of the mass $m_{L}$.

From Eq. (1), the natural frequency is

$$
f=\frac{1}{2 \pi} \sqrt{\frac{k}{M+m+m_{L} L^{2}}} .
$$

From the experimental results, the natural frequency of the lever is $5 \mathrm{~Hz}$. Thus, the spring constant of the measurement system can be given as follows:

\section{$k=(2 \pi f)^{2} \times\left(M+m+m_{L} L^{2}\right)=89.2 \times 10^{3}$.}

The damper coefficient can be adjusted so as to match the convergence rate in the responses of the lever.

From Eq. (1), the Roverbal displacement at steady-state for the open-loop system $(F=0)$ can be described by:

$\mathbf{x}=\frac{\boldsymbol{M g}}{\boldsymbol{k}}$.

In addition, the lever displacement at steady state, which is the Roverbal displacement magnified by the lever, can be obtained by the following equation:

$x_{L}=-L x$.

Simulations of the measurement system can be performed using Eq. (1)-(5). Figure 5 shows a block diagram of the

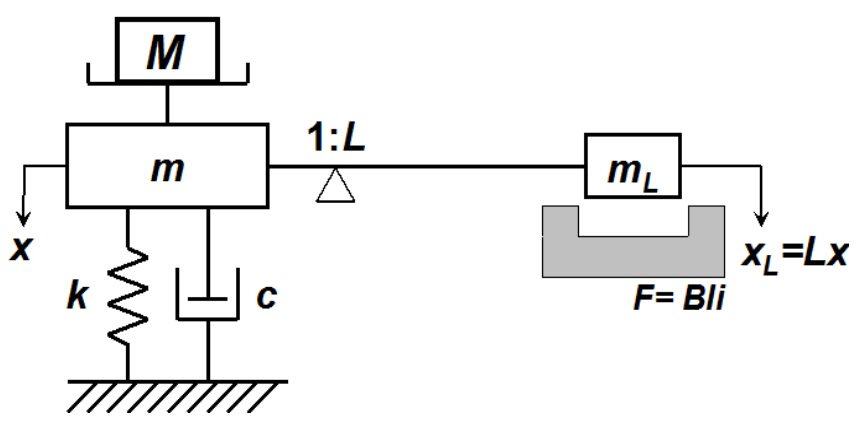

Figure 4. Physical model of measurement system. 


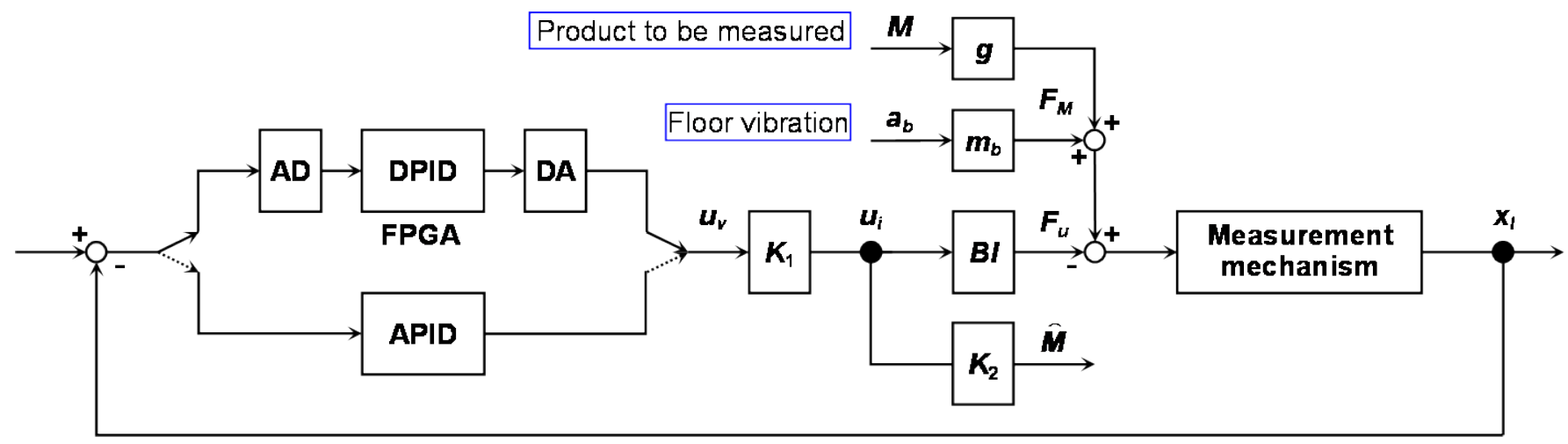

Figure 5. Block diagram of measurement system.

measurement system. In simulations and experiments, the validity of the model can be confirmed by comparing the sensor outputs of the lever displacement $\left(x_{I}\right)$.

\section{EXPERIMENTAL AND SIMULATION RESULTS FOR STEP RESPONSE}

In this section, the validity of the proposed model is confirmed by comparing the simulation result and the experimental results for open-loop and closed-loop systems.

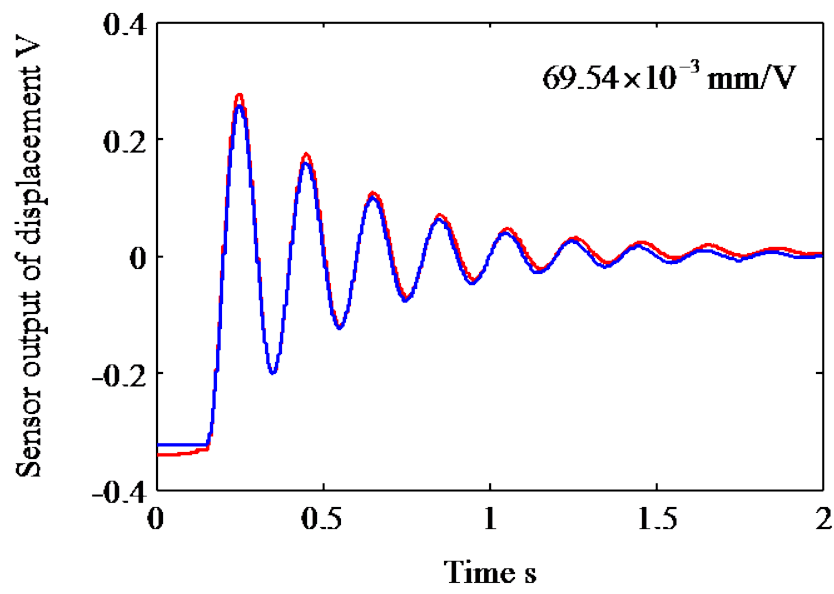

(a) $M=0.01 \mathrm{~kg}$

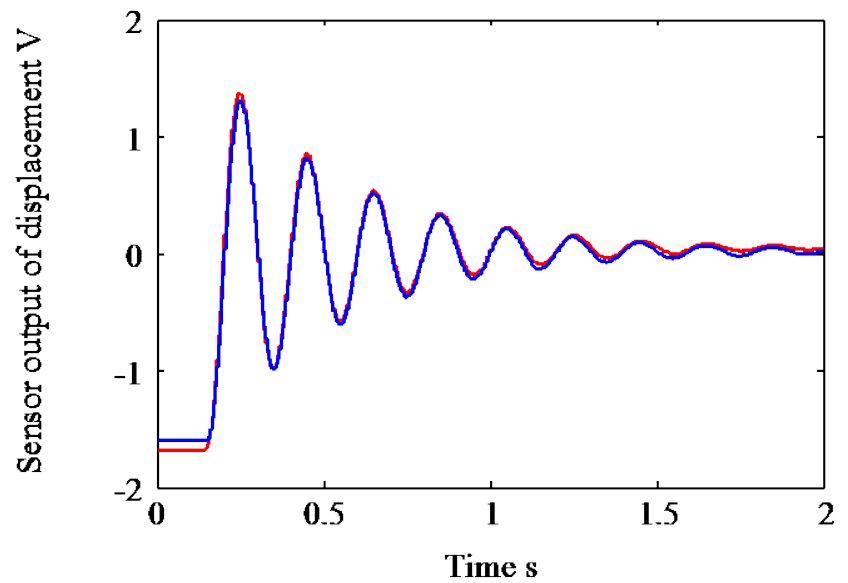

(c) $M=0.05 \mathrm{~kg}$

\subsection{Open-loop system}

The validity of the proposed model was first examined by comparing the simulation and experimental results for the open-loop system.

Figure 6 depicts the experimental and simulation results for $M=0.01,0.02,0.05$ and $0.1 \mathrm{~kg}$. The red and blue lines indicate the experimental and simulation results, respectively. The startup operation is the time when the product of the mass $M$ is put on the measurement system. When the time is $0.2 \mathrm{~s}$, the product of the mass $M$ is removed. At the same time, the

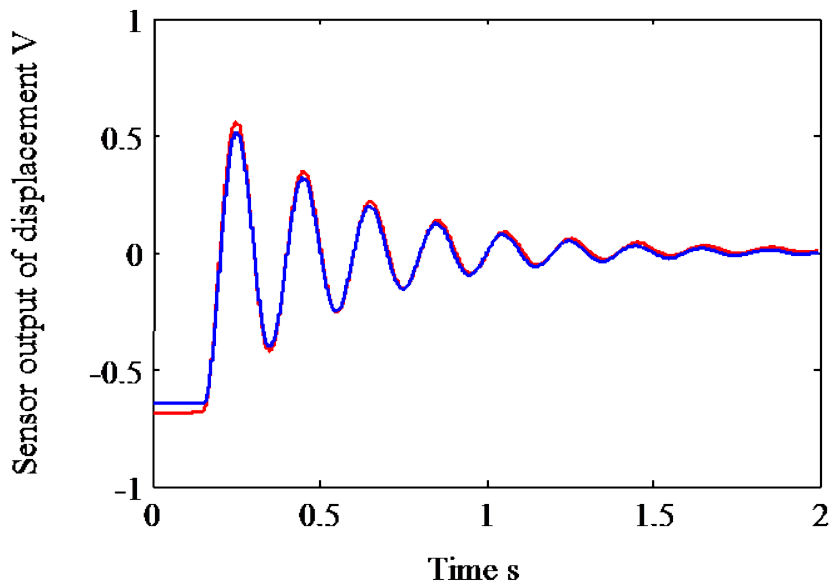

(b) $M=0.02 \mathrm{~kg}$

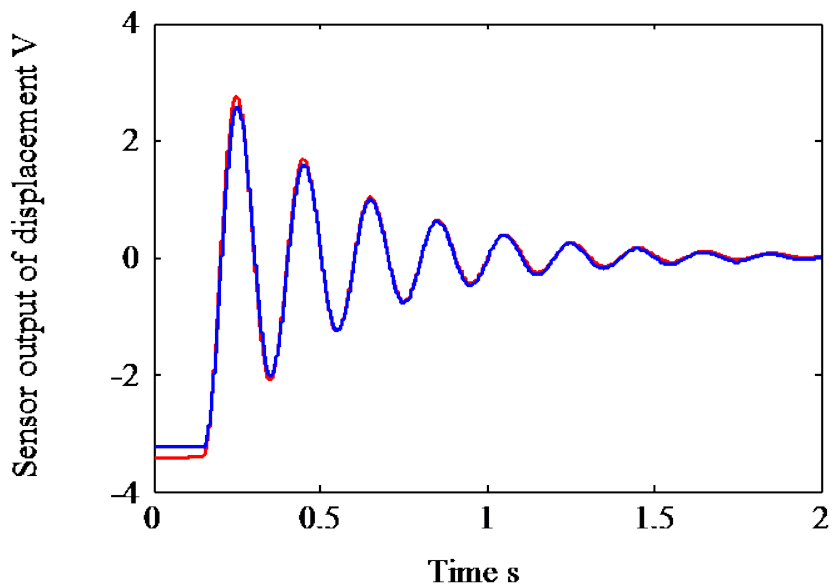

(d) $M=0.1 \mathrm{~kg}$

Figure 6. Comparison between experimental result and simulation result (open loop). 


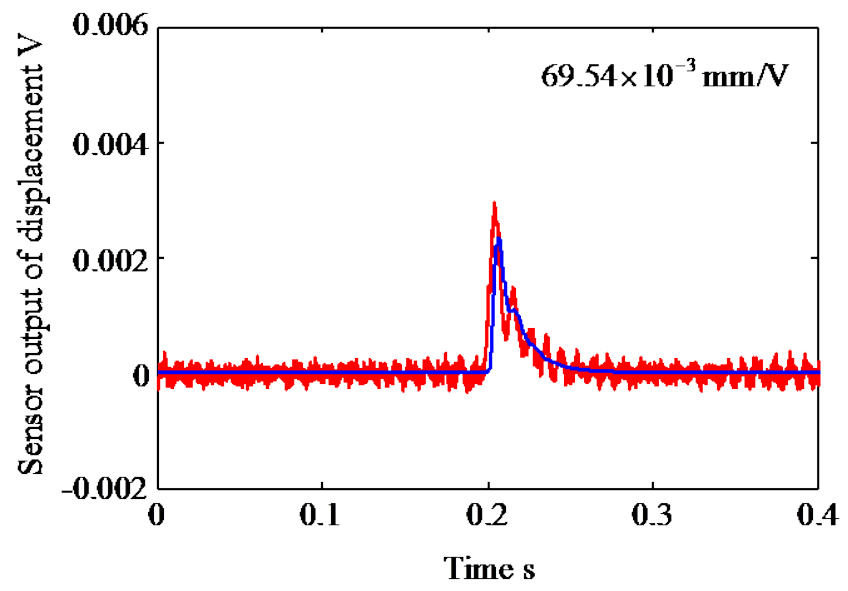

(a) $M=0.01 \mathrm{~kg}$

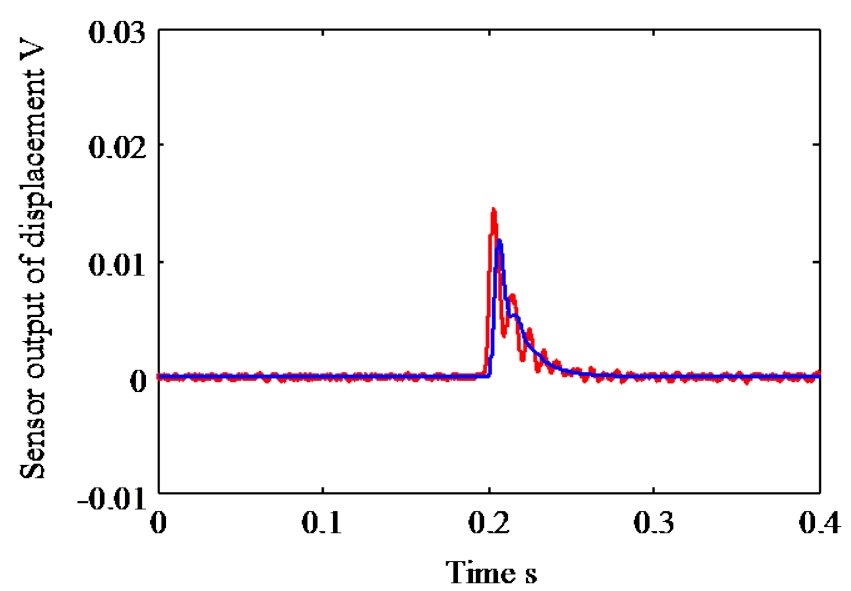

(c) $M=0.05 \mathrm{~kg}$

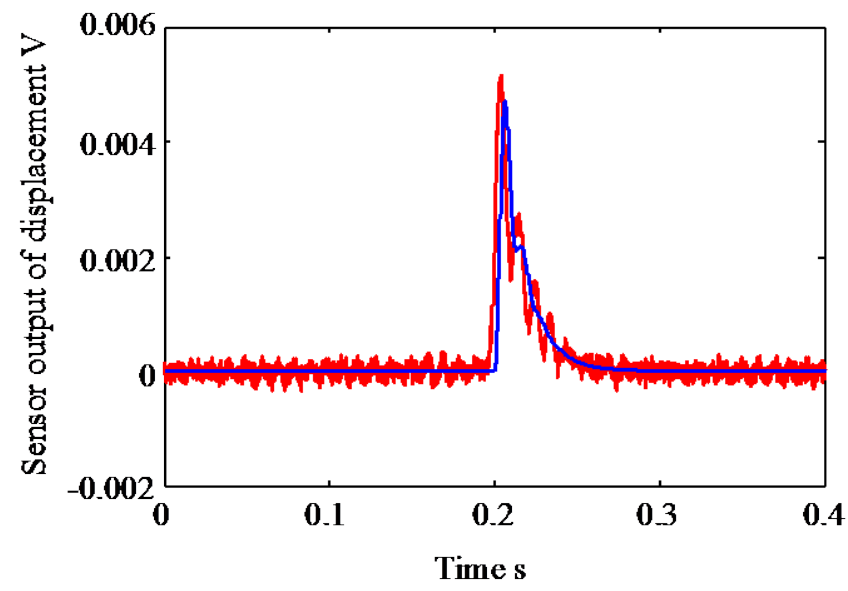

(b) $M=0.02 \mathrm{~kg}$

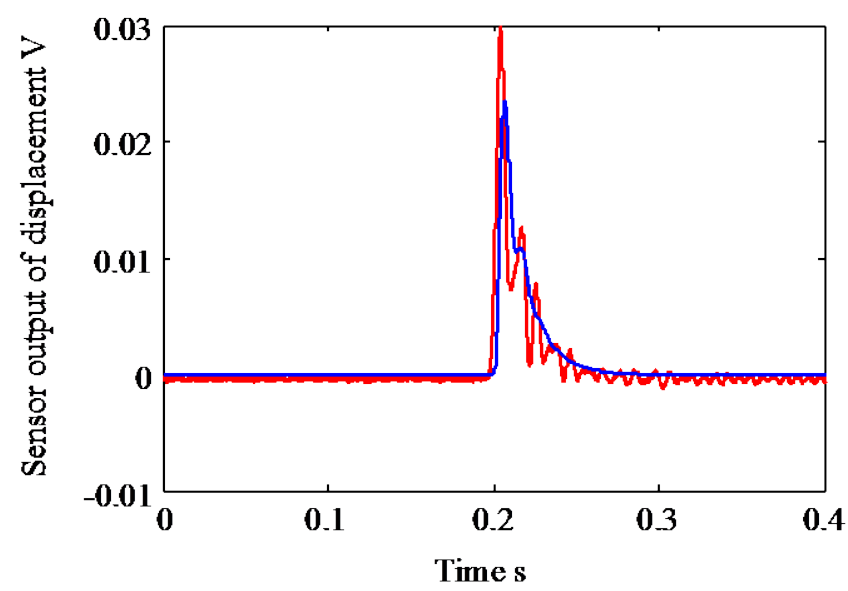

(d) $M=0.1 \mathrm{~kg}$

Figure 7. Comparison between experimental result and simulation result (closed loop).

displacement $\chi_{L}$ is measured by the displacement sensor. In Figure 6, the vertical axis shows the displacement sensor output of the lever displacement.

It can be seen from these figures that the responses of the simulation were in excellent agreement with the experimental results. Thus, the validity of the proposed model was confirmed for the open-loop system. Although the amplitude of the response changes depending on the mass, the convergence time is not changed. Thus, this measurement system can be assumed to be a linear system. Namely, even if the controller is added to the measurement system, the response does not depend on the mass.

In the next section, the validity of the proposed model for the closed-loop system was examined.

\subsection{Closed-loop system}

This section explains the experimental and simulation results with EMFC. The electromagnetic force was controlled by a proportional-integral-differential (PID) control scheme. Taking the actual circuit of the $\mathrm{D}$ action into account, the ideal D action could not be implemented. So, it is assumed that the D action is the approximated differential action. Namely, the transfer function of the PID controller $C(s)$ can be described by

$$
C(s)=k_{p}+\frac{k_{i}}{s}+\frac{k_{d} s}{k_{d t} s+1}
$$

where $k_{\phi}$ is the proportional gain, $k_{i}$ is the integral gain, and $k_{d n}$ and $k_{d d}$ are the numerator and denominator coefficients of the differential gains, respectively. The control voltage $U_{\nu}(s)$ (Laplace transform of $\left.u_{v}(t)\right)$ can be adjusted as follows:

$U_{\mathrm{v}}(\mathrm{s})=\mathrm{C}(\mathrm{s}) E(\mathrm{~s})$,

where $E(s)$ is Laplace transform of $e(t)$, and $e(t)\left(=x_{L r}-x_{L}\right)$ is the error between the reference $x_{L r}$ and the displacement sensor output of the lever $x_{L}$. The PID control can be performed by FPGA (Field Programmable Gate Array) every $0.1 \mathrm{~ms}$.

Figure 7 depicts the experimental and simulation results for $M=0.01,0.02,0.05$ and $0.1 \mathrm{~kg}$. The red and blue lines indicate the experimental and simulation results, respectively. The simulation and experimental conditions are the same as the open-loop system.

Responses such as the convergence time, the rise time and the settling time were almost the same for the simulation and experimental results. Thus, the validity of the proposed EMFC model was confirmed. We consider that the reasons for the modeling error were the frictional force in small displacements and the magnification mechanism of the lever. However, the peak value of the simulation result for $M=0.1 \mathrm{~kg}$ was different from that of the experimental result. Moreover, a highfrequency response occurred in the experiments due to the system noise. 


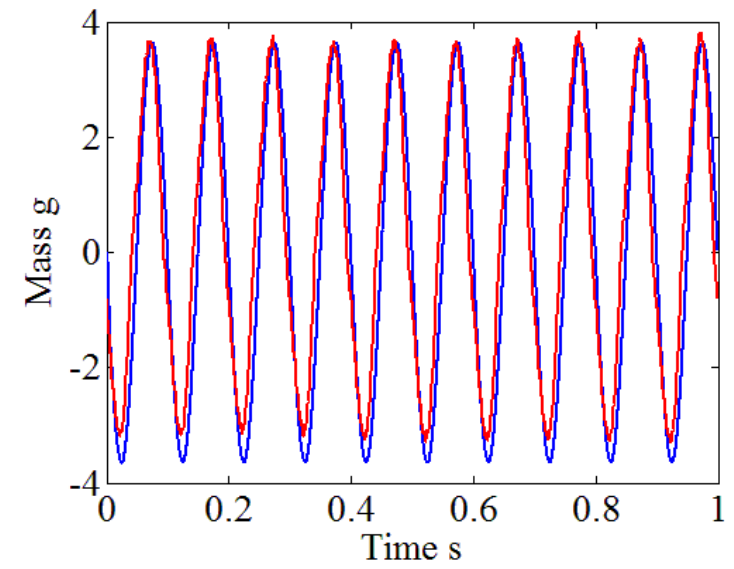

Figure 8. Simulation result of floor vibration (amplitude: $1 \mathrm{~mm}$, frequency: $10 \mathrm{~Hz}$ ).

\section{SIMULATION RESULTS OF FLOOR VIBRATION}

Here, we discuss the effect of floor vibrations in the simulation and the experimental results. The floor vibration oscillates the base of the system. The vibration input applies to the same position in the block diagram (Figure 5) as the mass of the object. In the experiment, a vibration exciter is used in order to oscillate the system.

Figure 8 shows the simulation result and the experimental result of floor vibration. The amplitude and the frequency of the vibration were set at $x_{b}=1 \mathrm{~mm}$ and $f_{b}=10 \mathrm{~Hz}$, respectively. The angular frequency $\omega_{b}$ can be calculated by $2 \pi f_{b}$. The mass $m_{b}$ of the base of the system was set at $0.175 \mathrm{~kg}$. The acceleration input $a_{b}$ of the floor vibration was set at $x_{b} \times \omega_{b}^{2}$. The red and blue lines indicate the experimental and simulation results, respectively. As can be seen from this result, exactly the same responses were obtained.

In addition, Figure 9 shows the results for the simulation and the experiment when the amplitude and frequency of the floor vibration were set at $x_{b}=1.25 \mathrm{~mm}$ and $f_{b}=10 \mathrm{~Hz}$, respectively. The red and blue lines indicate the experimental and simulation results, respectively. It can be seen from Figure 9 that the same responses were obtained. The validity of the proposed model was thus confirmed for different amplitudes of vibration.

Furthermore, Figure 10 shows the result of the simulation and the experiment in which the amplitude and the frequency

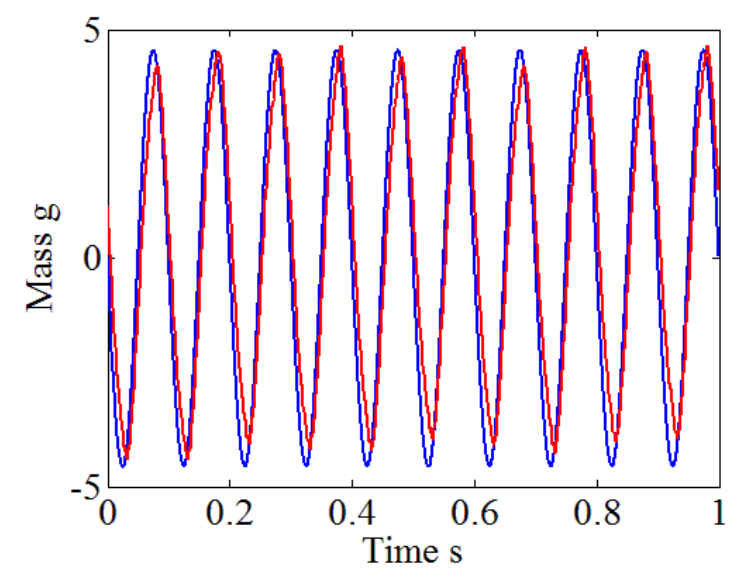

Figure 9. Simulation result of floor vibration (amplitude: $1.25 \mathrm{~mm}$, frequency: $10 \mathrm{~Hz}$ ).

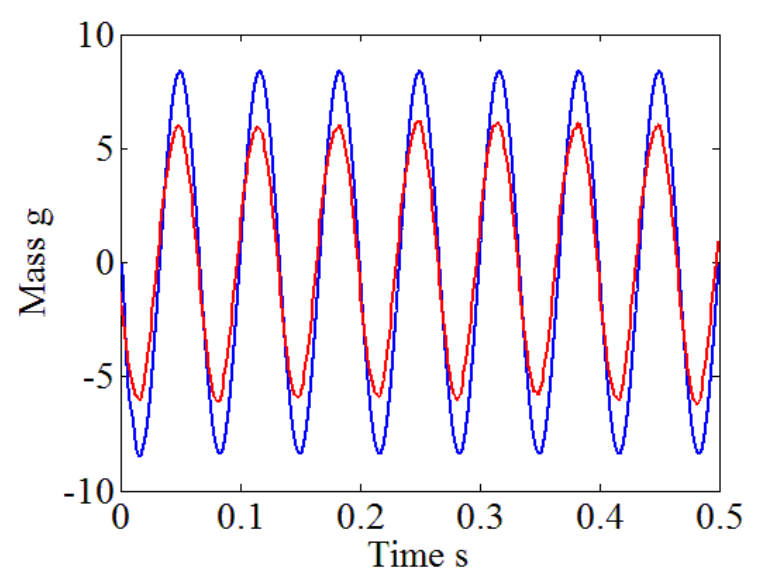

Figure 10. Simulation result of floor vibration (amplitude: $1 \mathrm{~mm}$, frequency: $15 \mathrm{~Hz}$ ).

of the floor vibration were set at $x_{b}=1 \mathrm{~mm}$ and $f_{b}=15 \mathrm{~Hz}$. The red and blue lines indicate the experimental and simulation results, respectively. The error between the simulation and the experimental result was about $1 \mathrm{~g}$. When the frequency of the floor vibration increases by 1.5 times, the acceleration increases $2.25\left(=1.5^{2}\right)$ times. Thus, the output theoretically increases 2.25 times in the simulation. However, the output of the experimental result increased only about 1.75 times in this case. We consider that the reason was the mechanical limitation of the displacement in the mass measurement system in order to avoid the system breakdown. The simulation could not replicate the experimental results for this reason.

As a result, we can evaluate the effect of the floor vibration by the proposed model.

\section{CONCLUSIONS}

In this paper, a dynamic model of an electromagnetic force compensation (EMFC) system was constructed. The model was approximated by a spring-mass-damper system. The model parameters were identified by the experimental data for an open-loop response.

Then, the validation of the proposed model was examined for both open-loop and closed-loop systems. The simulation and experimental results for the open-loop system were exactly the same. The results for the closed-loop system were almost the same. As a result, the validity of the proposed EMFC model could be confirmed. In addition, we confirmed the validity of the proposed model with simulations of floor vibrations.

In the future, an entirely new control scheme for the electromagnetic force compensation can be implemented using the proposed model. This high-speed, high-accuracy mass measurement system will be applicable to many industrial fields.

\section{REFERENCES}

[1] T. Ono, "Basic point of dynamic mass measurement", Proc. SICE, pp. 43-44, 1999.

[2] T. Ono, "Dynamic weighing of mass", Instrumentation and Automation 12, No. 2, pp. 35, 1984.

[3] W.G. Lee et al., "Development of speed and accuracy for mass measurements in checkweighers and conveyor belt scales", Proc. ISMFM, pp. 23-28, 1994.

[4] K. Kameoka et al., "Signal processing for checkweigher", Proc. APMF, pp. 122-128, 1996.

[5] Y. Yamakawa and T. Yamazaki, "Mathematical Model of Checkweigher with Electromagnetic Force Compensation", Proc. XX IMEKO World Congress, TC3-O-19, 2012. 